\section{A 'Solid-Image' Microscope}

For many purposes, such as tracing networks of neural structure in the brain, a normal microscope has the serious limitation that only a thin layer of the specimen can be studied at one time, owing to the limited depth of focus of microseope objectives. When a thin section is examined, only those structures which happen to lie in the plane of the section can be observed. This plane may be confused by such objects as fibres coming away from or toward the plane of the section. A microscope which gave a large depth of field, and which presented the image as a solid in a luminous block in which the structures could be observed in depth would have important advantages over existing optical microscopes. We have designed and constructed a primitive prototype of an instrument giving such a 'solid image'. It is possible to observe this from any position and to see the structures with appropriate parallax.

The instrument involves two processes. (1) The focal plane of the objective is effectively made to 'scan' up and down through the depth of the section. In the prototype instrument, this occurs at a rate of 50 double scans/sec. The slide is mounted on a steel fork tuned to $50 \mathrm{c} / \mathrm{s}$. which is driven by a polarized solenoid energized by a $50 \mathrm{c} . / \mathrm{s}$. sine wave. Thus the slide is carried up and down through the focal plane of the objective 50 times/sec. (2) The image is projected on to a screen mounted on a second tuned fork which vibrates at the same rate and in the same phase as the slide carrying the specimen.

Scanning serves to extract the information in depth from the specimen; the second process reconstitutes it in depth, giving a 'solid image' in the space swept by the screen. Its frequency of vibration is greater than the fusion frequency for the eye, so that little or no flicker is observed.

As the instrument stands at present, the resolution and contrast leave much to be desired. A test slide of human hair does, however, give a fairly satisfactory 'solid image' in which the positions of the hairs may be seen in depth. These positions change as the observer changes his viewing position. If the objective is brought farther from, or closer to, the slide the image of the test hairs moves through the luminous cube in a convincing manner. So far, magnifications greater than 100 have not been attempted, and the greatest useful magnification is only about 20.

We hope to produce an improved version, using the same basic principles but perhaps abandoning the physical screen in favour of shifting the virtual image of viewing lenses. The tuned forks may be replaced by some more precise system for obtaining the 'scanning' oscillatory movements. We intend to modulate the light intensity in order to increase the illumination inside the image. At present the front and back are brighter than the middle, because the velocity of the screen is greater at the centre of its sweep.

It is hoped that an instrument might be developed along these lines which will be useful for biological and other research purposes.

\section{R. L. Gregory}

Psychological Laboratory,

University of Cambridge.

P. E. K. Donaldson

Physiological Laboratory,

University of Cambridge.

Oct. 16.

\section{Evidence for ${ }_{2}^{6} \mathrm{He}$ 'Clusters' in Nuclei}

ThE well-known ' $\alpha$-particle' model of the nucleus, whereby certain low- $A$ nuclei are considered as consisting of groups of $\alpha$-particles, has recently received a new impetus as a result of work by Wildermuth and Kanellopoulos' on the 'cluster model' of nuclei. 'These authors have presented a formal treatment of the behaviour of 'cluster states' and illustrated it with particular reference to the nucleus ${ }_{4}^{8} \mathrm{Be}$ considered as two $\alpha$-particle clusters. At the same time they have pointed out that clusters other than ${ }_{2}^{4} \mathrm{He}$ might be present, and in fact suggested that stable closed shells such as those with neutron numbers, $N$, of 50 and 82 , could be considered as large clusters. The question now arises as to which elusters are in fact suitable representations of particular nucleonic configurations, and the purpose of this communication is to present evidence in this connexion.

This evidence is obtained from a simple study of the neutron numbers, $N$, and atomic numbers, $Z$, of stable nuclei. If clusters are in fact entities inside the nucleus, one would expect the $(N-Z)$ systematics of stable nuclei to show a tendency for stable nuclei to be separated from each other by the sub. nuclear clusters. That such tendencies do in fact exist can be seen from the published work of one of $u_{s^{2,3}}$, and we present here further evidence by way of illustration. Fig. $I$ is an $(N-Z)$ versus $Z$ diagram on which the stable even- $Z$ odd- $N$ nuclides have been plotted. The following features emerge very clearly: (a) In the low- $Z$ region the stable nuclides are separated from each other by steps of ${ }_{2}^{4} \mathrm{He}$. (b) For higher- $Z(44-56)$ the separation is in steps of ${ }_{2}^{6} \mathrm{He}$ and not ${ }_{2}^{4} \mathrm{He}$ (c) The sequences are interrupted by the 'magic numbers'.

It should be mentioned that the region $Z$ 16-44 has been omitted in order to preserve clarity in presentation. In most of this region separations of both ${ }_{2}^{4} \mathrm{He}$ and ${ }_{2}^{6} \mathrm{He}$ appear--it may therefore be regarded as some sort of intermediate region.

If now the ${ }_{2}^{4} \mathrm{He}$ steps in the low- $Z$ region are taken as evidence of the presence of ${ }_{2}^{4} \mathrm{He}$ clusters in low. mass nuclei (as indeed seems reasonable), then it appears likely that ${ }_{2}^{6} \mathrm{He}$ clusters are present in certain nuclei of higher mass. The same effect obtains, although not so strikingly and with less clarity, if one plots stable odd-oven or even-odd nuclides (see, for example, ref. 3, Fig. 3, and ref. 2, Fig. 19).

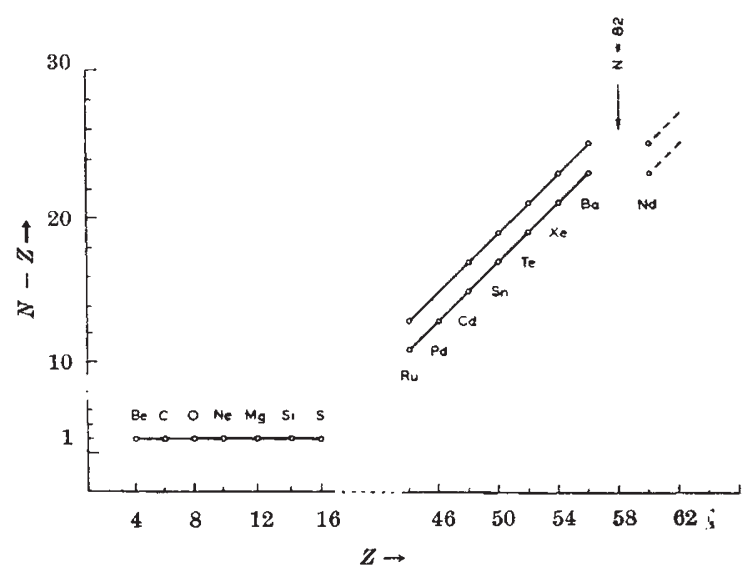

Fig. 1. $(N-Z)$ vs. $Z$ diagram of stable even- $Z$, odd- $N$ nuclides The 'missing' nuclide ${ }_{4}^{102} \mathrm{Pd}$ is very long lived with a half-life of $7 \times 10^{6}$ years 